\title{
MAX-STABLE PROCESSES AND THE FUNCTIONAL $D$-NORM REVISITED
}

\author{
STEFAN AULBACH, MICHAEL FALK, MARTIN HOFMANN AND MAXIMILIAN ZOTT \\ The final publication is available at Springer via \\ http: //dx. doi.org/10.1007/s10687-014-0210-0
}

\begin{abstract}
Aulbach et al. (2013) introduced a max-domain of attraction approach for extreme value theory in $C[0,1]$ based on functional distribution functions, which is more general than the approach based on weak convergence in de Haan and Lin (2001). We characterize this new approach by decomposing a process into its univariate margins and its copula process. In particular, those processes with a polynomial rate of convergence towards a max-stable process are considered. Furthermore we investigate the concept of differentiability in distribution of a max-stable processes.
\end{abstract}

\section{INTRODUCTION}

A stochastic process $\boldsymbol{\xi}=\left(\xi_{t}\right)_{t \in[0,1]}$ on the interval $[0,1]$, whose sample paths belong to the space $C[0,1]$ of continuous functions on $[0,1]$, is called max-stable (MSP), if there are norming functions $a_{n}, b_{n} \in C[0,1], a_{n}>0$, such that the distribution of the process $\max _{1 \leq i \leq n}\left(\boldsymbol{\xi}^{(i)}-b_{n}\right) / a_{n}$ coincides with that of $\boldsymbol{\xi}$ for each $n \in \mathbb{N}$. By $\boldsymbol{\xi}^{(1)}, \boldsymbol{\xi}^{(2)}, \ldots$ we denote independent copies of $\boldsymbol{\xi}$.

Aulbach et al. (2013) established a characterization of the distribution of an MSP via a norm on $E[0,1]$, the space of bounded functions on $[0,1]$ which have finitely many discontinuities. This norm is called $D$-norm and it is defined by means of a so-called generator process.

An MSP $\boldsymbol{\eta}=\left(\eta_{t}\right)_{t \in[0,1]} \in C[0,1]$ with standard negative exponential margins $P\left(\eta_{t} \leq x\right)=\exp (x), x \leq 0$, will be called a standard max-stable process (SMSP). As the distribution of a stochastic process on $[0,1]$ is determined by its finite dimensional marginal distributions, a process $\boldsymbol{\eta} \in C[0,1]$ with identical marginal distribution function (df) $F(x)=\exp (x), x \leq 0$, is standard max-stable if and only

Key words and phrases. Max-stable process, D-norm, functional max-domain of attraction, copula process, generalized Pareto process, $\delta$-neighborhood of generalized Pareto process, derivative of $D$-norm, distributional differentiability. 
if

$$
P\left(\boldsymbol{\eta} \leq \frac{f}{n}\right)^{n}=P(\boldsymbol{\eta} \leq f), \quad f \in E^{-}[0,1], n \in \mathbb{N},
$$

where $E^{-}[0,1]$ denotes the subset of those functions in $E[0,1]$, which attain only non positive values. Note that it would be sufficient in equation (11) to consider $f \in$ $C^{-}[0,1]$ of non positive continuous functions. The extension to $E^{-}[0,1]$, however, provides the inclusion of the finite dimensional marginal distributions (fidis) of $\boldsymbol{\eta}$, as

$$
P\left(\eta_{t_{i}} \leq x_{i}, 1 \leq i \leq d\right)=P(\boldsymbol{\eta} \leq f)
$$

where $0 \leq t_{1}<\cdots<t_{d} \leq 1$ and $f \in E^{-}[0,1]$ is given by $f\left(t_{i}\right)=x_{i}<0$ for $i \in\{1, \ldots, d\}$ and $f(t)=0$ for $t \in[0,1] \backslash\left\{t_{1}, \ldots, t_{d}\right\}$.

From Aulbach et al. (2013) we know that (11) is equivalent with the condition that there is some norm $\|\cdot\|_{D}$ on $E[0,1]$, called $D$-norm, satisfying

$$
P(\boldsymbol{\eta} \leq f)=\exp \left(-\|f\|_{D}\right), \quad f \in E^{-}[0,1] .
$$

Precisely, there exists a stochastic process $\boldsymbol{Z}=\left(Z_{t}\right)_{t \in[0,1]} \in C[0,1]$ with

$$
0 \leq Z_{t} \leq m, \quad E\left(Z_{t}\right)=1, \quad t \in[0,1],
$$

for some number $m \geq 1$, such that

$$
\|f\|_{D}=E\left(\sup _{t \in[0,1]}\left(|f(t)| Z_{t}\right)\right), \quad f \in E[0,1] .
$$

The condition $Z_{t} \leq m, t \in[0,1]$, can be weakened to $E\left(\sup _{t \in[0,1]} Z_{t}\right)<\infty$. Observe that property (2) of a max-stable process $\boldsymbol{\eta}$ with standard negative exponential margins corresponds to the spectral representation of a max-stable process with unit Fréchet margins as considered in Resnick and Rov (1991) and de Haan (1984) $)$, since $P\left(-\eta_{t}^{-1} \leq y\right)=\exp \left(-y^{-1}\right)$ for $y>0$ and $t \in[0,1]$.

Based on this characterization, Aulbach et al. (2013) introduced a functional domain of attraction approach for stochastic processes in terms of convergence of their distribution functions. This functional domain of attraction is larger than the one based on weak convergence as investigated in de Haan and Lin (2001), cf. Aulbach et al. (2013, Proposition 5). In Section 2 of the present paper we will carry over de Haan and Lin's (2001) characterization of max-domain of attraction for stochastic processes in $C[0,1]$ in terms of weak convergence to our domain of attraction approach based on convergence of df.

Buishand et al. (2008) suggested the definition of generalized Pareto processes (GPP), which extends the multivariate Peaks-Over-Threshold approach to function spaces. This particular approach was investigated and settled in Ferreira and 
de Haan (2012), Aulbach et al. (2013) and Dombry and Ribatet (2013). In Section 3 we will introduce certain $\delta$-neighborhoods of GPP, which can be characterized by their rate of convergence towards a max-stable process. This is in complete accordance with the multivariate case.

Finally, we establish the concept of differentiability in distribution of an SMSP in Section 4. To this end, we investigate some properties of SMSP such as the partial derivatives of a $D$-norm, the distribution of the increments of an SMSP and the conditional distribution of an SMSP given one point being observed.

To improve the readability of this paper we use bold face such as $\boldsymbol{\xi}, \boldsymbol{X}$ for stochastic processes and default font $f, a_{n}$ etc. for non stochastic functions. Operations on functions such as $\boldsymbol{\xi}<f$ or $\left(\boldsymbol{\xi}-b_{n}\right) / a_{n}$ are meant pointwise. The usual abbreviations iid, a.s. and $r v$ for the terms independent and identically distributed, almost surely and random variable, respectively, are used.

\section{A Characterization of MaX-Domain of Attraction}

In the multivariate framework, it is well-known that a $\operatorname{rv}\left(X_{1}, \ldots, X_{d}\right)$ is in the domain of attraction of a multivariate max-stable distribution if and only if its copula has this property and the distribution of $X_{i}$ is in the univariate domain of attraction of a max-stable distribution for each $i=1, \ldots, n$. We refer to Galambos (1978), Deheuvels (1978, 1984) and Aulbach et al. (2012) for details.

De Haan and Lin (2001) extended this result to the usual weak convergence of the maximum of $n$ iid stochastic processes, linearly standarized, towards a maxstable process in $C[0,1]$. They provided a condition which entails a characterization of the domain of attraction property in the sense of usual weak convergence in $C[0,1]$ of stochastic processes. This condition is essentially condition (3) below. The characterization of the domain of attraction property then consists of uniform weak convergence on $[0,1]$ of the marginal maxima to a univariate extreme value distribution, together with weak convergence of the corresponding copula process in function space. We will carry over de Haan and Lin's (2001) result to our domain of attraction approach based on convergence of dfs of stochastic processes, see Theorem 2.1 and Corollary 2.2 below. As established in Aulbach et al. (2013, Proposition 5), the max-domain of attraction we consider is larger than that based on ordinary weak convergence. But it is still an open problem whether it is strictly larger.

Let $\boldsymbol{X}=\left(X_{t}\right)_{t \in[0,1]} \in C[0,1]$ be a stochastic process with continuous marginal df $F_{t}(x)=P\left(X_{t} \leq x\right), x \in \mathbb{R}, t \in[0,1]$, and let $\boldsymbol{\xi}=\left(\xi_{t}\right)_{t \in[0,1]} \in C[0,1]$ be an MSP with marginal df $G_{t}, t \in[0,1]$. Suppose that there exist norming functions 
$a_{n}, b_{n} \in C[0,1], a_{n}>0, n \in \mathbb{N}$, such that

$$
\sup _{t \in[0,1]}\left|n\left(F_{t}\left(a_{n}(t) f(t)+b_{n}(t)\right)-1\right)-\log \left(G_{t}(f(t))\right)\right| \rightarrow_{n \rightarrow \infty} 0
$$

for each $f \in E[0,1]$ with $\inf _{t \in[0,1]} G_{t}(f(t))>0$. This is essentially condition (3.11) in de Haan and Lin (2001). Using Taylor expansion $\log (1+\varepsilon)=\varepsilon+O\left(\varepsilon^{2}\right)$ as $\varepsilon \rightarrow 0$, condition (3) in particular implies weak convergence of the univariate margins

$$
F_{t}\left(a_{n}(t) x+b_{n}(t)\right)^{n} \rightarrow_{n \rightarrow \infty} G_{t}(x), \quad x \in \mathbb{R}, t \in[0,1] .
$$

Put $\boldsymbol{U}:=\left(U_{t}\right)_{t \in[0,1]}:=\left(F_{t}\left(X_{t}\right)\right)_{t \in[0,1]}$, which is the copula process corresponding to $\boldsymbol{X}$. Let $\boldsymbol{U}^{(1)}, \boldsymbol{U}^{(2)}, \ldots$ be independent copies of $\boldsymbol{U}$, and let $\boldsymbol{X}^{(1)}, \boldsymbol{X}^{(2)}, \ldots$ be independent copies of $\boldsymbol{X}$. The following theorem is the main result of this section.

For the implication (4) $\Longrightarrow$ (5) we set $\eta_{t}:=\log \left(G_{t}\left(\xi_{t}\right)\right), t \in[0,1]$, and for the reverse conclusion $\xi_{t}:=G_{t}^{-1}\left(\exp \left(\eta_{t}\right)\right), t \in[0,1]$. In both cases the processes $\boldsymbol{\xi}:=\left(\xi_{t}\right)_{t \in[0,1]}$ and $\boldsymbol{\eta}:=\left(\eta_{t}\right)_{t \in[0,1]} \in C[0,1]$ are max-stable, $\boldsymbol{\eta}$ being an SMSP. By Lemma 1 in Aulbach et al. (2013) or the elementary arguments as in the proof of Theorem 9.4.1 in de Haan and Ferreira (2006), one obtains that $P\left(G_{t}\left(\xi_{t}\right)=\right.$ 0 for some $t \in[0,1])=0$, i.e., the processes $\boldsymbol{\eta}$ and $\boldsymbol{\xi}$ are well defined.

Theorem 2.1. We have under condition (3)

$$
P\left(\max _{1 \leq i \leq n} \frac{\boldsymbol{X}^{(i)}-b_{n}}{a_{n}} \leq f\right) \rightarrow_{n \rightarrow \infty} P(\boldsymbol{\xi} \leq f), \quad f \in E[0,1],
$$

if and only if

$$
P\left(n \max _{1 \leq i \leq n}\left(\boldsymbol{U}^{(i)}-1\right) \leq g\right) \rightarrow_{n \rightarrow \infty} P(\boldsymbol{\eta} \leq g), \quad g \in E^{-}[0,1] .
$$

Proof. As $\boldsymbol{\xi}$ has continuous sample paths and $G_{t}$ is continuous for each $t \in[0,1]$, we have continuity of the function $[0,1] \ni t \mapsto G_{t}(x)$ uniformly for $x \in \mathbb{R}$. Thus

$$
\left|G_{t}(x)-G_{t_{0}}\left(x_{0}\right)\right| \leq\left|G_{t}(x)-G_{t_{0}}(x)\right|+\left|G_{t_{0}}(x)-G_{t_{0}}\left(x_{0}\right)\right|
$$

implies the continuity of the function $[0,1] \times \mathbb{R} \ni(t, x) \mapsto G_{t}(x)$. This shows in particular that any discontinuity of $[0,1] \ni t \mapsto G_{t}(h(t))$ is also a discontinuity of $h \in E[0,1]$. The same arguments apply to the function $[0,1] \times \mathbb{R} \ni(t, x) \mapsto F_{t}(x)$.

We first establish the implication (4) $\Longrightarrow$ (5). Choose $g \in E^{-}[0,1]$ with $\sup _{t \in[0,1]} g(t)<0$ and put $f(t):=G_{t}^{-1}(\exp (g(t))$. Then $f \in E[0,1]$ and we obtain from assumption (4)

$$
P\left(\max _{1 \leq i \leq n} \boldsymbol{X}^{(i)} \leq a_{n} f+b_{n}\right) \rightarrow_{n \rightarrow \infty} P(\boldsymbol{\xi} \leq f)=P(\boldsymbol{\eta} \leq g)=\exp \left(-\|g\|_{D}\right),
$$

where $\|\cdot\|_{D}$ is the $D$-norm corresponding to the SMSP $\boldsymbol{\eta}$. 
We have, on the other hand, by condition (3) and the continuity of the marginal df $F_{t}$

$$
\begin{aligned}
& P\left(\max _{1 \leq i \leq n} \boldsymbol{X}^{(i)} \leq a_{n} f+b_{n}\right) \\
& =P\left(n \max _{1 \leq i \leq n}\left(U_{t}^{(i)}-1\right) \leq n\left(F_{t}\left(a_{n}(t) f(t)+b_{n}(t)\right)-1\right), t \in[0,1]\right) \\
& =P\left(n \max _{1 \leq i \leq n}\left(U_{t}^{(i)}-1\right) \leq g(t)+r_{n}(t), t \in[0,1]\right),
\end{aligned}
$$

where $r_{n}(t)=o(1)$ as $n \rightarrow \infty$, uniformly for $t \in[0,1]$. As an intermediate step we claim that

$$
P\left(n \max _{1 \leq i \leq n}\left(\boldsymbol{U}^{(i)}-1\right) \leq g\right) \rightarrow_{n \rightarrow \infty} P(\boldsymbol{\eta} \leq g), \quad g \in E^{-}[0,1], \sup _{t \in[0,1]} g(t)<0 .
$$

Replace $g$ by $g+\varepsilon$ and $g-\varepsilon$ for $\varepsilon>0$ small enough such that $g+\varepsilon<0$, and put

$$
f_{\varepsilon}(t):=G_{t}^{-1}(\exp (g(t)+\varepsilon)), \quad f_{-\varepsilon}(t):=G_{t}^{-1}(\exp (g(t)-\varepsilon)), \quad t \in[0,1] .
$$

Then $f_{\varepsilon}, f_{-\varepsilon} \in E[0,1]$, and we obtain from condition (3) and equation (6) for $n \geq n_{0}$

$$
\begin{aligned}
& P\left(n \max _{1 \leq i \leq n}\left(U_{t}^{(i)}-1\right) \leq n\left(F_{t}\left(a_{n}(t) f_{\varepsilon}(t)+b_{n}(t)\right)-1\right), t \in[0,1]\right) \\
& \geq P\left(n \max _{1 \leq i \leq n}\left(\boldsymbol{U}^{(i)}-1\right) \leq g\right) \\
& \geq P\left(n \max _{1 \leq i \leq n}\left(U_{t}^{(i)}-1\right) \leq n\left(F_{t}\left(a_{n}(t) f_{-\varepsilon}(t)+b_{n}(t)\right)-1\right), t \in[0,1]\right),
\end{aligned}
$$

where the upper bound converges to $\exp \left(-\|g+\varepsilon\|_{D}\right)$ and the lower bound to $\exp \left(-\|g-\varepsilon\|_{D}\right)$. As both converge to $\exp \left(-\|g\|_{D}\right)$ as $\varepsilon \rightarrow 0$, we have established (77).

Next we claim that (7) is true for each $g \in E^{-}[0,1]$, i.e., we drop the assumption $\sup _{t \in[0,1]} g(t)<0$. From (17) we deduce that for each $\varepsilon>0$

$$
\lim _{n \rightarrow \infty} P\left(n \max _{1 \leq i \leq n}\left(\boldsymbol{U}^{(i)}-1\right) \leq g-\varepsilon\right)=\exp \left(-\|g-\varepsilon\|_{D}\right)
$$

and, thus,

$$
\begin{aligned}
\liminf _{n \rightarrow \infty} P\left(n \max _{1 \leq i \leq n}\left(\boldsymbol{U}^{(i)}-1\right) \leq g\right) & \geq \lim _{n \rightarrow \infty} P\left(n \max _{1 \leq i \leq n}\left(\boldsymbol{U}^{(i)}-1\right) \leq g-\varepsilon\right) \\
& =\exp \left(-\|g-\varepsilon\|_{D}\right) .
\end{aligned}
$$

As $\varepsilon>0$ was arbitrary, we have established that

$$
\liminf _{n \rightarrow \infty} P\left(n \max _{1 \leq i \leq n}\left(\boldsymbol{U}^{(i)}-1\right) \leq g\right) \geq \exp \left(-\|g\|_{D}\right) .
$$


On the other hand, we have by (7) for $\varepsilon>0$

$$
\lim _{n \rightarrow \infty} P\left(n \max _{1 \leq i \leq n}\left(\boldsymbol{U}^{(i)}-1\right) \leq-\varepsilon\right)=\exp \left(-\varepsilon\|1\|_{D}\right) \rightarrow_{\varepsilon \downarrow 0} 1,
$$

and, thus,

$$
\begin{aligned}
& \limsup _{n \rightarrow \infty} P\left(n \max _{1 \leq i \leq n}\left(\boldsymbol{U}^{(i)}-1\right) \leq g\right) \\
& \leq \limsup _{n \rightarrow \infty}\left(P\left(n \max _{1 \leq i \leq n}\left(\boldsymbol{U}^{(i)}-1\right) \leq g, n \max _{1 \leq i \leq n}\left(\boldsymbol{U}^{(i)}-1\right) \leq-\varepsilon\right)\right. \\
& \left.+P\left(\left(n \max _{1 \leq i \leq n}\left(\boldsymbol{U}^{(i)}-1\right) \leq-\varepsilon\right)^{\complement}\right)\right) \\
& =\exp \left(-\|\left(\min (g(t),-\varepsilon)_{t \in[0,1]} \|_{D}\right)+1-\exp \left(-\varepsilon\|1\|_{D}\right)\right.
\end{aligned}
$$

by (7). As the first term in the final line above converges to $\exp \left(-\|g\|_{D}\right)$ as $\varepsilon \downarrow 0$ and the second one to zero, we have established that

$$
\limsup _{n \rightarrow \infty} P\left(n \max _{1 \leq i \leq n}\left(\boldsymbol{U}^{(i)}-1\right) \leq g\right) \leq \exp \left(-\|g\|_{D}\right) .
$$

This proves equation (7) for arbitrary $g \in E^{-}[0,1]$ and completes the proof of the conclusion (4) $\Longrightarrow$ (5).

Next we establish the implication (5) $\Longrightarrow$ (4). Choose $f \in E[0,1]$ with $\inf _{t \in[0,1]} G_{t}(f(t))>0$ and put $g(t):=\log \left(G_{t}(f(t))\right), t \in[0,1]$. From the assumption (5) we obtain

$$
P\left(n \max _{1 \leq i \leq n}\left(\boldsymbol{U}^{(i)}-1\right) \leq g\right) \rightarrow_{n \rightarrow \infty} P(\boldsymbol{\eta} \leq g)=P(\boldsymbol{\xi} \leq f)=\exp \left(-\|g\|_{D}\right) .
$$

On the other hand, we have by condition (3)

$$
\begin{aligned}
& P\left(n \max _{1 \leq i \leq n}\left(\boldsymbol{U}^{(i)}-1\right) \leq g\right) \\
& =P\left(n \max _{1 \leq i \leq n}\left(\boldsymbol{U}_{t}^{(i)}-1\right) \leq n\left(F_{t}\left(a_{n}(t) f(t)+b_{n}(t)\right)-1\right)+r_{n}(t), t \in[0,1]\right),
\end{aligned}
$$

where $r_{n}(t)=o(1)$ as $n \rightarrow \infty$, uniformly for $t \in[0,1]$. We claim that

$$
\begin{aligned}
& P\left(n \max _{1 \leq i \leq n}\left(\boldsymbol{U}_{t}^{(i)}-1\right) \leq n\left(F_{t}\left(a_{n}(t) f(t)+b_{n}(t)\right)-1\right), t \in[0,1]\right) \\
& \rightarrow_{n \rightarrow \infty} P(\boldsymbol{\eta} \leq g)=\exp \left(-\|g\|_{D}\right) .
\end{aligned}
$$

Replace $g$ by $\min (g+\varepsilon, 0)$ and $g-\varepsilon$, where $\varepsilon>0$ is arbitrary. Then we obtain from (5) and condition (3) for $n \geq n_{0}=n_{0}(\varepsilon)$

$$
\begin{aligned}
& P\left(n \max _{1 \leq i \leq n}\left(\boldsymbol{U}^{(i)}-1\right) \leq \min (g+\varepsilon, 0)\right) \\
& =P\left(n \max _{1 \leq i \leq n}\left(\boldsymbol{U}^{(i)}-1\right) \leq g+\varepsilon\right)
\end{aligned}
$$




$$
\begin{aligned}
& \geq P\left(n \max _{1 \leq i \leq n}\left(\boldsymbol{U}^{(i)}-1\right) \leq n\left(F_{t}\left(a_{n}(t) f(t)+b_{n}(t)\right)-1\right), t \in[0,1]\right) \\
& \geq P\left(n \max _{1 \leq i \leq n}\left(\boldsymbol{U}^{(i)}-1\right) \leq g-\varepsilon\right) .
\end{aligned}
$$

Since

$$
P\left(n \max _{1 \leq i \leq n}\left(\boldsymbol{U}^{(i)}-1\right) \leq \min (g+\varepsilon, 0)\right) \rightarrow_{n \rightarrow \infty} \exp \left(-\|\min (g+\varepsilon, 0)\|_{D}\right)
$$

as well as

$$
P\left(n \max _{1 \leq i \leq n}\left(\boldsymbol{U}^{(i)}-1\right) \leq g-\varepsilon\right) \rightarrow_{n \rightarrow \infty} \exp \left(-\|g-\varepsilon\|_{D}\right)
$$

and $\varepsilon>0$ was arbitrary, (8) follows.

From the equality

$$
\begin{aligned}
& P\left(n \max _{1 \leq i \leq n}\left(\boldsymbol{U}^{(i)}-1\right) \leq n\left(F_{t}\left(a_{n}(t) f(t)+b_{n}(t)\right)-1\right), t \in[0,1]\right) \\
& =P\left(\max _{1 \leq i \leq n} \boldsymbol{X}^{(i)} \leq a_{n} f+b_{n}\right)
\end{aligned}
$$

and from (8) we obtain

$$
\lim _{n \rightarrow \infty} P\left(\max _{1 \leq i \leq n} \boldsymbol{X}^{(i)} \leq a_{n} f+b_{n}\right)=P(\boldsymbol{\xi} \leq f)
$$

for each $f \in E[0,1]$ with $\inf _{t \in[0,1]} G_{t}(f(t))>0$. If $\inf _{t \in[0,1]} G_{t}(f(t))=0$, then, for $\varepsilon>0$, there exists $t_{0} \in[0,1]$ such that $G_{t_{0}}\left(f\left(t_{0}\right)\right) \leq \varepsilon$. We, thus, have $P(\boldsymbol{\xi} \leq f) \leq$ $P\left(\xi_{t_{0}} \leq f\left(t_{0}\right)\right)=G_{t_{0}}\left(f\left(t_{0}\right)\right) \leq \varepsilon$ and, by condition (3),

$$
\begin{aligned}
P\left(\max _{1 \leq i \leq n} \boldsymbol{X}^{(i)} \leq a_{n} f+b_{n}\right) & \leq P\left(\max _{1 \leq i \leq n} X_{t_{0}}^{(i)} \leq a_{n}\left(t_{0}\right) f\left(t_{0}\right)+b_{n}\left(t_{0}\right)\right) \\
& \rightarrow n \rightarrow \infty G_{t_{0}}\left(f\left(t_{0}\right)\right) \leq \varepsilon .
\end{aligned}
$$

As $\varepsilon>0$ was arbitrary, we have established

$$
\lim _{n \rightarrow \infty} P\left(\max _{1 \leq i \leq n} \boldsymbol{X}^{(i)} \leq a_{n} f+b_{n}\right)=0=P(\boldsymbol{\xi} \leq f)
$$

in that case, where $\inf _{t \in[0,1]} G_{t}(f(t))=0$ and, thus, (9) for each $f \in E[0,1]$. This completes the proof of Theorem 2.1]

We say that a stochastic process $\boldsymbol{X}=\left(X_{t}\right)_{t \in[0,1]} \in C[0,1]$ is in the domain of attraction of a max-stable process $\boldsymbol{\xi}=\left(\xi_{t}\right)_{t \in[0,1]} \in C[0,1]$, denoted by $\boldsymbol{X} \in \mathcal{D}(\boldsymbol{\xi})$, if there are norming functions $a_{n}>0, b_{n} \in C[0,1], n \in \mathbb{N}$, such that (4) holds. The following consequence of Theorem 2.1 extends the well-known characterization of multivariate domain of attraction in terms of weak convergence of the univariate margins together with weak convergence of the copulas (Galambos (1978). Deheuvels (1978, 1984) and Aulbach et al. (2012)) to the functional space. 
Corollary 2.2. Let $\boldsymbol{X}=\left(X_{t}\right)_{t \in[0,1]} \in C[0,1]$ be a stochastic process with identical continuous marginal df $F(x)=P\left(X_{t} \leq x\right), x \in \mathbb{R}, t \in[0,1]$, and let $\boldsymbol{\xi}=\left(\xi_{t}\right)_{t \in[0,1]} \in$ $C[0,1]$ be an MSP with identical marginal $d f G$. Denote by $\boldsymbol{U}=\left(U_{t}\right)_{t \in[0,1]}:=$ $\left(F\left(X_{t}\right)\right)_{t \in[0,1]}$ the copula process pertaining to $\boldsymbol{X}$. Then we have $\boldsymbol{X} \in \mathcal{D}(\boldsymbol{\xi})$ if and only if $\boldsymbol{U} \in \mathcal{D}(\boldsymbol{\eta})$ and $F \in \mathcal{D}(G)$.

For a characterization of the condition $\boldsymbol{U} \in \mathcal{D}(\boldsymbol{\eta})$ in terms of certain neighborhoods of generalized Pareto processes see Proposition 3.4 below.

Proof of Corollary 2.2. The assumption $F \in \mathcal{D}(G)$ yields $\sup _{x \in \mathbb{R}} \mid F^{n}\left(a_{n} x+b_{n}\right)-$ $G(x) \mid \rightarrow_{n \rightarrow \infty} 0$ for some sequence of norming constants $a_{n}>0, b_{n} \in \mathbb{R}, n \in \mathbb{N}$. Taking logarithms and using Taylor expansion of $\log (1+G(x))$ for $x \in\left[x_{0}, x_{1}\right]$ with $G\left(x_{0}\right)>0$ implies

$$
\sup _{x \in\left[x_{0}, x_{1}\right]}\left|n\left(F\left(a_{n} x+b_{n}\right)-1\right)-\log (G(x))\right| \rightarrow_{n \rightarrow \infty} 0
$$

and, thus, condition (3) is satisfied. Corollary 2.2 is now an immediate consequence of Theorem 2.1 together with the fact that the assumption $\boldsymbol{X} \in \mathcal{D}(\boldsymbol{\xi})$ implies in particular that $F \in \mathcal{D}(G)$.

We conclude this section with a short remark. Choose $f \in E[0,1]$ which is not the constant function zero. If $\boldsymbol{\eta} \in C^{-}[0,1]$ is an SMSP, then the univariate rv

$$
\eta_{f}:=\sup _{t \in[0,1]} \frac{\eta_{t}}{|f(t)|}
$$

is by equation (2) negative exponential distributed with parameter $\|f\|_{D}$. This explains why $P\left(\sup _{t \in[0,1]} \eta_{t}<0\right)=1$.

\section{3. $\delta$-Neighborhood of a Generalized Pareto Process}

First we recall some facts from Aulbach et al. (2013). A univariate generalized Pareto distribution (GPD) $W$ is simply given by $W(x)=1+\log (G(x)), G(x) \geq 1 / e$, where $G$ is a univariate extreme value distribution (EVD). It was established by Pickands (1975) and Balkema and de Haan (1974) that, roughly, the maximum of $n$ iid univariate observations, linearly standardized, converges in distribution to an EVD as $n$ increases if, and only if, the exceedances above an increasing threshold follow a generalized Pareto distribution (GPD). The multivariate extension is due to Rootzén and Tajvidi (2006).

Theorem 2.2.5 in Falk et al. (2011) characterizes $\delta$-neighborhoods of univariate GPD via of a polynomial rate of convergence of the maximum in an iid sample towards its limiting extreme value distribution. The multivariate extension is given 
in Theorem 5.5.5 in Falk et al. (2011). In Proposition 3.7 we will extend this characterization of a polynomial rate of convergence to function space. We, therefore, have to introduce generalized Pareto processes and their spectral $\delta$-neighborhoods first.

It was observed by Buishand et al. (2008) that a $d$-dimensional GPD $W$ with ultimately standard Pareto margins can be represented in its upper tail as $W(\boldsymbol{x})=$ $P\left(U^{-1} \boldsymbol{Z} \leq \boldsymbol{x}\right), \mathbf{0} \leq \boldsymbol{x}_{0} \leq \boldsymbol{x} \in \mathbb{R}^{d}$, where the rv $U$ is uniformly on $(0,1)$ distributed and independent of the $\operatorname{rv} \boldsymbol{Z}=\left(Z_{1}, \ldots, Z_{d}\right)$ with $0 \leq Z_{i} \leq m$ for some $m \geq 1$ and $E\left(Z_{i}\right)=1,1 \leq i \leq d$. The following definition extends this approach to function spaces.

Definition 3.1. Let $U$ be a rv which is uniformly distributed on $[0,1]$ and independent of the generator process $Z \in C[0,1]$ that is characterized by the two properties

$$
0 \leq Z_{t} \leq m \quad \text { and } \quad E\left(Z_{t}\right)=1, \quad t \in[0,1],
$$

for some constant $m \geq 1$. Then the stochastic process

$$
\boldsymbol{Y}:=\frac{1}{U} \boldsymbol{Z} \in C^{+}[0,1]
$$

is a generalized Pareto process (GPP) with ultimately standard Pareto margins Buishand et al. (2008).

Corollary 9.4.5 in de Haan and Ferreira (2006) implies that one can choose the generator process $\boldsymbol{Z}$ such that $\sup _{t \in[0,1]} Z_{t}=$ const a.s.

Characterizations of GPP in terms of a functional Peaks-Over-Threshold approach are established in Ferreira and de Haan (2012) and Dombry and Ribatet (2013). Local asymptotic normality in $\delta$-neighborhoods of standard generalized Pareto processes was established in Aulbach and Falk (2012a), and tests for a generalized Pareto process are investigated in Aulbach and Falk (2012b).

The univariate margins $Y_{t}$ of $\boldsymbol{Y}$ have ultimately standard Pareto tails:

$$
\begin{aligned}
P\left(Y_{t} \leq x\right) & =P\left(\frac{1}{x} Z_{t} \leq U\right) \\
& =\int_{0}^{m} P\left(\frac{1}{x} z \leq U\right)\left(P * Z_{t}\right)(d z) \\
& =1-\frac{1}{x} \int_{0}^{m} z\left(P * Z_{t}\right)(d z) \\
& =1-\frac{1}{x} E\left(Z_{t}\right) \\
& =1-\frac{1}{x}, \quad x \geq m, 0 \leq t \leq 1,
\end{aligned}
$$

where $P * Z_{t}$ denotes the distribution of $Z_{t}$. 
Put $\boldsymbol{V}:=\max (-1 / \boldsymbol{Y}, M)$, where $M<0$ is an arbitrary constant, which ensures that $\boldsymbol{V}>-\infty$. Then, as shown in Example 1 in Aulbach et al. (2013),

$$
P(\boldsymbol{V} \leq f)=P\left(\sup _{t \in[0,1]}\left(|f(t)| Z_{t}\right) \leq U\right)=1-\|f\|_{D}
$$

for all $f \in E^{-}[0,1]$ with $\|f\|_{\infty} \leq \min (1 / m,|M|)$, i.e., $\boldsymbol{V}$ has the property that its functional $\mathrm{df}$ is in its upper tail equal to

$$
W(f):=P(\boldsymbol{V} \leq f)=1+\log (G(f)), \quad f \in E^{-}[0,1],\|f\|_{\infty} \leq \min (1 / m,|M|),
$$

where $G(f)=P(\boldsymbol{\eta} \leq f)$ is the functional df of the MSP $\boldsymbol{\eta}$ with $D$-norm $\|\cdot\|_{D}$ and generator $\boldsymbol{Z}$. This representation of the upper tail of a GPP in terms of $1+\log (G)$ is in complete accordance with the uni- and multivariate case (see, for example, Falk et al., 2011, Chapter 5). We write $W=1+\log (G)$ in short notation and call $\boldsymbol{V}$ a GPP as well, this time with ultimately uniform margins.

REMARK 3.2. Due to representation (10), the GPP $\boldsymbol{V}$ is obviously in the functional domain of attraction of an SMSP $\boldsymbol{\eta}$ with $D$-norm $\|\cdot\|_{D}$ and generator $\boldsymbol{Z}$ : Take $a_{n}=1 / n$ and $b_{n}=0$. We have for $f \in E^{-}[0,1]$ and large enough $n \in \mathbb{N}$

$$
P\left(\boldsymbol{V} \leq \frac{1}{n} f\right)^{n}=\left(1-\frac{1}{n}\|f\|_{D}\right)^{n} \rightarrow_{n \rightarrow \infty} \exp \left(-\|f\|_{D}\right)=P(\boldsymbol{\eta} \leq f) .
$$

The following result is a functional version of the well-known fact that the spectral df of a GPD rv is equal to a uniform df in a neighborhood of zero.

Lemma 3.3. We have for $f \in E^{-}[0,1]$ with $0<\|f\|_{\infty} \leq m$ and some $s_{0}<0$

$$
W_{f}(s):=P(\boldsymbol{V} \leq s|f|)=1+s\|f\|_{D}, \quad s_{0} \leq s \leq 0 .
$$

The next result is established in Aulbach et al. (2013). Let $\boldsymbol{U}$ be a copula process and let $\boldsymbol{\eta}$ be an SMSP with functional df $P(\boldsymbol{\eta} \leq f)=\exp \left(-\|f\|_{D}\right), f \in E^{-}[0,1]$.

Proposition 3.4. The property $\boldsymbol{U} \in \mathcal{D}(\boldsymbol{\eta})$ is equivalent to the condition

$$
\lim _{s \uparrow 0} \frac{1-H_{f}(s)}{1-W_{f}(s)}=1, \quad f \in E^{-}[0,1],
$$

i.e., the spectral df $H_{f}(s)=P(\boldsymbol{U}-1 \leq s|f|), s \leq 0$, of $\boldsymbol{U}-1$ is for each $f \in E^{-}[0,1]$ with $\|f\|_{\infty}>0$ tail equivalent to that of the GPD $W_{f}(s)=1+s\|f\|_{D}, s_{0} \leq s \leq 0$.

This characterization of the domain of attraction of an SMSP in terms of a certain GPP suggests to focus on the following standard case. Recall that Section 2 justified to consider SMSPs in place of general MSPs. 
Definition 3.5. A stochastic process $\boldsymbol{V} \in C^{-}[0,1]$ is a standard generalized Pareto process (SGPP), if there exists a $D$-norm $\|\cdot\|_{D}$ on $E[0,1]$ and some $c_{0}>0$ such that

$$
P(\boldsymbol{V} \leq f)=1-\|f\|_{D}
$$

for all $f \in E^{-}[0,1]$ with $\|f\|_{\infty} \leq c_{0}$.

The same arguments as at the end of Section 2 provide the upper tail of the df of the rv

$$
V_{f}:=\sup _{t \in[0,1]} \frac{V_{t}}{|f(t)|}
$$

if $\boldsymbol{V} \in C^{-}[0,1]$ is an SGPP and $f \in E[0,1]$ is not the constant function zero. We obtain

$$
P\left(V_{f}>x\right)=\|f\|_{D}|x|, \quad-1 \leq x \leq 0,
$$

if $\|f\|_{\infty} \leq c_{0}$, i.e., $V_{f}$ follows in its upper tail, precisely on $(-1,0)$, a uniform distribution.

Using the spectral decomposition of a stochastic process in $C^{-}[0,1]$, we can easily extend the definition of a spectral $\delta$-neighborhood of a multivariate GPD as in Falk et al. (2011, Section 5.5) to the spectral $\delta$-neighborhood of an SGPP. Denote by $E_{1}^{-}[0,1]=\left\{f \in E^{-}[0,1]:\|f\|_{\infty}=1\right\}$ the unit sphere in $E^{-}[0,1]$.

Definition 3.6. We say that a stochastic process $\boldsymbol{Y} \in C^{-}[0,1]$ belongs to the spectral $\delta$-neighborhood of the SGPP $\boldsymbol{V}$ for some $\delta \in(0,1]$, if we have uniformly for $f \in E_{1}^{-}[0,1]$ the expansion

$$
\begin{aligned}
1-P(\boldsymbol{Y} \leq c f) & =(1-P(\boldsymbol{V} \leq c f))\left(1+O\left(c^{\delta}\right)\right) \\
& =c\|f\|_{D}\left(1+O\left(c^{\delta}\right)\right)
\end{aligned}
$$

as $c \downarrow 0$.

An SMSP is, for example, in the spectral $\delta$-neighborhood of the corresponding GPP with $\delta=1$.

The following result extends Theorem 5.5.5 in Falk et al. (2011) on the rate of convergence of multivariate extremes. It shows that $\delta$-neighborhoods collect, roughly, all processes which have a polynomial rate of convergence towards an SMSP.

Proposition 3.7. Let $\boldsymbol{Y}$ be a stochastic process in $C^{-}[0,1], \boldsymbol{V}$ an $S G P P$ with $D$-norm $\|\cdot\|_{D}$ and $\boldsymbol{\eta}$ an $S M S P$ with $P(\boldsymbol{\eta} \leq f)=\exp \left(-\|f\|_{D}\right), f \in E^{-}[0,1]$. 
(i) Suppose that $\boldsymbol{Y}$ is in the spectral $\delta$-neighborhood of $\boldsymbol{V}$ for some $\delta \in(0,1]$. Then we have

$$
\sup _{f \in E^{-}[0,1]}\left|P\left(\boldsymbol{Y} \leq \frac{f}{n}\right)^{n}-P(\boldsymbol{\eta} \leq f)\right|=O\left(n^{-\delta}\right) .
$$

(ii) Suppose that $H_{f}(c)=P(\boldsymbol{Y} \leq c|f|)$ is differentiable with respect to $c$ in a left neighborhood of 0 for any $f \in E_{1}^{-}[0,1]$, i.e., $h_{f}(c):=(\partial / \partial c) H_{f}(c)$ exists for $c \in(-\varepsilon, 0)$ and any $f \in E_{1}^{-}[0,1]$. Suppose, moreover, that $H_{f}$ satisfies the von Mises condition

$$
\frac{-c h_{f}(c)}{1-H_{f}(c)}=: 1+r_{f}(c) \rightarrow_{c \uparrow 0} 1, \quad f \in E_{1}^{-}[0,1],
$$

with remainder term $r_{f}$ satisfying

$$
\sup _{f \in E_{1}^{-}[0,1]}\left|\int_{c}^{0} \frac{r_{f}(t)}{t} d t\right| \rightarrow_{c \uparrow 0} 0 .
$$

If

$$
\sup _{f \in E^{-}[0,1]}\left|P\left(\boldsymbol{Y} \leq \frac{f}{n}\right)^{n}-P(\boldsymbol{\eta} \leq f)\right|=O\left(n^{-\delta}\right)
$$

for some $\delta \in(0,1]$, then $\boldsymbol{Y}$ is in the spectral $\delta$-neighborhood of the GPP $V$.

Proof. Note that

$$
\begin{aligned}
& \sup _{f \in E^{-}[0,1]}\left|P\left(\boldsymbol{Y} \leq \frac{f}{n}\right)^{n}-P(\boldsymbol{\eta} \leq f)\right| \\
= & \sup _{f \in E^{-}[0,1]}\left|P\left(\boldsymbol{Y} \leq \frac{\|f\|_{\infty}}{n} \frac{f}{\|f\|_{\infty}}\right)^{n}-P\left(\boldsymbol{\eta} \leq\|f\|_{\infty} \frac{f}{\|f\|_{\infty}}\right)\right| \\
= & \sup _{c<0} \sup _{f \in E_{1}^{-}[0,1]}\left|P\left(\boldsymbol{Y} \leq \frac{c}{n}|f|\right)^{n}-P(\boldsymbol{\eta} \leq c|f|)\right| \\
= & \sup _{f \in E_{1}^{-}[0,1]} \sup _{c<0}\left|P\left(\boldsymbol{Y} \leq \frac{c}{n}|f|\right)^{n}-P(\boldsymbol{\eta} \leq c|f|)\right| .
\end{aligned}
$$

The assertion now follows by repeating the arguments in the proof of Theorem 1.1 in Falk and Reiss (2002), where the bivariate case has been established.

\section{Distributional Differentiability of an SMSP}

In this section, our aim is to establish the concept of distributional differentiability of an SMSP $\boldsymbol{\eta}=\left(\eta_{t}\right)_{t \in[0,1]}$. In Proposition 4.4 we will prove the following result: Let $\boldsymbol{Z}=\left(Z_{t}\right)_{t \in[0,1]} \in C[0,1]$ be a generator process of $\boldsymbol{\eta}$. Suppose that $Z_{t}^{\prime}=(\partial / \partial t) Z_{t}$ exists for $t=t_{0}$ a.s. Then $\left(\eta_{t_{0}+h}-\eta_{t_{0}}\right) / h$ converges in distribution to some rv on the real line as $h \rightarrow 0$ and we compute its df. 
This is a first result on differentiability of max-stable processes. To the best of our knowledge, the question, under which conditions a max-stable process is differentiable at $t_{0} \in[0,1]$ a.s., is an open problem. If $\eta_{t}^{\prime}=(\partial / \partial t) \eta_{t}$ actually exists at $t=t_{0}$ a.s., the distribution of $\eta_{t_{0}}^{\prime}$ equals that of $-\eta_{t_{0}} \zeta_{t_{0}}$, where the rv $\zeta_{t_{0}}$ is independent of $\eta_{t_{0}}$ and has df $\mathbb{F}_{t_{0}}(x)=E\left(1_{\left\{Z_{t_{0}}^{\prime} \leq x Z_{t_{0}}\right\}} Z_{t_{0}}\right), x \in \mathbb{R}$; see the discussion after Proposition 4.4

As an auxiliary result, we first compute the partial derivatives of a functional $D$ norm $\|\cdot\|_{D}$. For this purpose, we need the following definition. Let $\mathcal{X}$ be a normed function space, and $J: \mathcal{X} \rightarrow \mathbb{R}$ a functional. The first variation (or the Gâteaux differential) of $J$ at $u \in \mathcal{X}$ in the direction $v \in \mathcal{X}$ is defined as

$$
\nabla J(u)(v):=\lim _{\varepsilon \rightarrow 0} \frac{J(u+\varepsilon v)-J(u)}{\varepsilon}=\left.\frac{d}{d \varepsilon} J(u+\varepsilon v)\right|_{\varepsilon=0} .
$$

Moreover, the right-hand (left-hand) first variation of $J$ at $u$ in the direction $v$ is defined as

$$
\nabla^{+} J(u)(v):=\lim _{\varepsilon \downarrow 0} \frac{J(u+\varepsilon v)-J(u)}{\varepsilon} \quad \text { and } \quad \nabla^{-} J(u)(v):=\lim _{\varepsilon \downarrow 0} \frac{J(u)-J(u-\varepsilon v)}{\varepsilon} .
$$

Considering a $D$-norm $\|\cdot\|_{D}$ a functional on the space $E[0,1]$, we can calculate the first variation of $\|\cdot\|_{D}$. The choice of the space $E[0,1]$ allows us the incorporation of the fidis and therefore yields the partial derivatives of a multivariate $D$-norm. This finite-dimensional version of the following result has already been observed by Einmahl et al. (2012). Note that as a norm is a convex function, a multivariate $D$-norm $\|\boldsymbol{x}\|_{D}$ is for almost every $\boldsymbol{x}<\mathbf{0} \in \mathbb{R}^{d}$ continuously differentiable.

Lemma 4.1. Let $\|\cdot\|_{D}$ be a D-norm on the function space $E[0,1]$ with generator $\boldsymbol{Z}=\left(Z_{t}\right)_{t \in[0,1]} \in C[0,1]$. Let $t_{0} \in[0,1]$ and $1_{\left\{t_{0}\right\}} \in E[0,1]$ be the indicator function of the one point set $\left\{t_{0}\right\}$. Then for every $f \in E[0,1]$

$$
\begin{aligned}
\nabla^{+}\|f\|_{D}\left(1_{\left\{t_{0}\right\}}\right) & =\lim _{\varepsilon \downarrow 0} \frac{\left\|f+\varepsilon 1_{\left\{t_{0}\right\}}\right\|_{D}-\|f\|_{D}}{\varepsilon} \\
& = \begin{cases}-E\left(1_{\left\{\sup _{t \neq t_{0}}|f(t)| Z_{t}<\left|f\left(t_{0}\right)\right| Z_{\left.t_{0}\right\}} Z_{t_{0}}\right),}\right. & f\left(t_{0}\right)<0, \\
+E\left(1_{\left\{\sup _{t \neq t_{0}}|f(t)| Z_{t} \leq\left|f\left(t_{0}\right)\right| Z_{t_{0}}\right\}} Z_{t_{0}}\right), & f\left(t_{0}\right) \geq 0,\end{cases}
\end{aligned}
$$

and

$$
\begin{aligned}
\nabla^{-}\|f\|_{D}\left(1_{\left\{t_{0}\right\}}\right) & =\lim _{\varepsilon \downarrow 0} \frac{\|f\|_{D}-\left\|f-\varepsilon 1_{\left\{t_{0}\right\}}\right\|_{D}}{\varepsilon} \\
& = \begin{cases}-E\left(1_{\left\{\sup _{t \neq t_{0}}|f(t)| Z_{t} \leq\left|f\left(t_{0}\right)\right| Z_{\left.t_{0}\right\}} Z_{t_{0}}\right),}\right. & f\left(t_{0}\right) \leq 0, \\
+E\left(1_{\left\{\sup _{t \neq t_{0}}|f(t)| Z_{t}<\left|f\left(t_{0}\right)\right| Z_{t_{0}}\right\}} Z_{t_{0}}\right), & f\left(t_{0}\right)>0 .\end{cases}
\end{aligned}
$$


Proof. Let $f \in E[0,1]$ with $f\left(t_{0}\right)>0$. We have

$$
\|f\|_{D}=E\left(\sup _{t \in[0,1]}|f(t)| Z_{t}\right) .
$$

First we calculate the right-hand first variation of $\|\cdot\|_{D}$, assuming $f\left(t_{0}\right) \geq 0$. For $\varepsilon>0$ there exists a disjoint decomposition of the underlying probability space $(\Omega, \mathcal{A}, P)$ via

$$
\Omega=A_{1}+A_{2}^{\varepsilon}+A_{3}^{\varepsilon}
$$

with

$$
\begin{aligned}
A_{1}: & =\left\{\sup _{t \neq t_{0}}|f(t)| Z_{t} \leq f\left(t_{0}\right) Z_{t_{0}}=\sup _{t \in[0,1]}|f(t)| Z_{t}\right\}, \\
A_{2}^{\varepsilon}: & =\left\{f\left(t_{0}\right) Z_{t_{0}}<\sup _{t \neq t_{0}}|f(t)| Z_{t}=\sup _{t \in[0,1]}|f(t)| Z_{t} \leq\left(f\left(t_{0}\right)+\varepsilon\right) Z_{t_{0}}\right\} \downarrow_{\varepsilon \downarrow 0} \emptyset \\
A_{3}^{\varepsilon}: & =\left\{\left(f\left(t_{0}\right)+\varepsilon\right) Z_{t_{0}}<\sup _{t \neq t_{0}}|f(t)| Z_{t}=\sup _{t \in[0,1]}|f(t)| Z_{t}\right\} .
\end{aligned}
$$

Therefore we obtain by the dominated convergence theorem

$$
\begin{aligned}
\nabla^{+} & \|f\|_{D}\left(1_{\left\{t_{0}\right\}}\right)=\lim _{\varepsilon \downarrow 0} \frac{\left\|f+\varepsilon 1_{\left\{t_{0}\right\}}\right\|_{D}-\|f\|_{D}}{\varepsilon} \\
= & \lim _{\varepsilon \downarrow 0} E\left(\frac{1}{\varepsilon}\left(\max \left(\sup _{t \neq t_{0}}|f(t)| Z_{t},\left(f\left(t_{0}\right)+\varepsilon\right) Z_{t_{0}}\right)-\sup _{t \in[0,1]}|f(t)| Z_{t}\right)\right) \\
= & \lim _{\varepsilon \downarrow 0} E\left(\frac{1}{\varepsilon}\left(\left(f\left(t_{0}\right)+\varepsilon\right) Z_{t_{0}}-f\left(t_{0}\right) Z_{t_{0}}\right) \cdot 1_{A_{1}}\right) \\
& +\lim _{\varepsilon \downarrow 0} E\left(\frac{1}{\varepsilon}\left(\left(f\left(t_{0}\right)+\varepsilon\right) Z_{t_{0}}-\sup _{t \neq t_{0}}|f(t)| Z_{t}\right) \cdot 1_{A_{2}^{\varepsilon}}\right) \\
& +\lim _{\varepsilon \downarrow 0} E\left(\frac{1}{\varepsilon}\left(\sup _{t \neq t_{0}}|f(t)| Z_{t}-\sup _{t \neq t_{0}}|f(t)| Z_{t}\right) \cdot 1_{A_{3}^{\varepsilon}}\right) \\
= & E\left(Z_{t_{0}} \cdot 1_{A_{1}}\right)
\end{aligned}
$$

since the second summand after the second to last equality vanishes as

$$
\begin{aligned}
\frac{1}{\varepsilon}\left(\left(f\left(t_{0}\right)+\varepsilon\right) Z_{t_{0}}-\sup _{t \neq t_{0}}|f(t)| Z_{t}\right) \cdot 1_{A_{2}^{\varepsilon}} & <\frac{1}{\varepsilon}\left(\left(f\left(t_{0}\right)+\varepsilon\right) Z_{t_{0}}-f\left(t_{0}\right) Z_{t_{0}}\right) \cdot 1_{A_{2}^{\varepsilon}} \\
& \rightarrow_{\varepsilon \downarrow \emptyset} Z_{t_{0}} \cdot 1_{\emptyset}=0 .
\end{aligned}
$$

Note that

$$
\sup _{t \neq t_{0}}|f(t)| Z_{t} \leq f\left(t_{0}\right) Z_{t_{0}} \Longleftrightarrow \sup _{t \in[0,1]}|f(t)| Z_{t}=f\left(t_{0}\right) Z_{t_{0}}
$$


The case $f\left(t_{0}\right) \leq 0$ works similarly. In order to calculate the left-side first variation of $\|\cdot\|_{D}$, assuming $f\left(t_{0}\right)>0$, we find for $\varepsilon>0$ a disjoint decomposition of $(\Omega, \mathcal{A}, P)$ via

$$
\Omega=B_{1}^{\varepsilon}+B_{2}^{\varepsilon}+B_{3}
$$

with

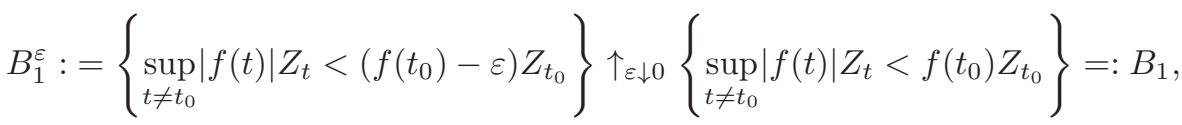

$$
\begin{aligned}
& B_{2}^{\varepsilon}:=\left\{\left(f\left(t_{0}\right)-\varepsilon\right) Z_{t_{0}} \leq \sup _{t \neq t_{0}}|f(t)| Z_{t}<\left(f\left(t_{0}\right)\right) Z_{t_{0}}\right\} \downarrow_{\varepsilon \downarrow 0}, \emptyset \\
& B_{3}:=\left\{f\left(t_{0}\right) Z_{t_{0}} \leq \sup _{t \neq t_{0}}|f(t)| Z_{t}\right\} \text {. }
\end{aligned}
$$

Hence we obtain again by the dominated convergence theorem

$$
\begin{aligned}
\nabla^{-} & \|f\|_{D}\left(1_{\left\{t_{0}\right\}}\right)=\lim _{\varepsilon \downarrow 0} \frac{\|f\|_{D}-\left\|f-\varepsilon 1_{\left\{t_{0}\right\}}\right\|_{D}}{\varepsilon} \\
= & \lim _{\varepsilon \downarrow 0} E\left(\frac{1}{\varepsilon}\left(\sup _{t \in[0,1]}|f(t)| Z_{t}-\max \left(\sup _{t \neq t_{0}}|f(t)| Z_{t},\left(f\left(t_{0}\right)-\varepsilon\right) Z_{t_{0}}\right)\right)\right) \\
= & \lim _{\varepsilon \downarrow 0} E\left(\frac{1}{\varepsilon}\left(f\left(t_{0}\right) Z_{t_{0}}-\left(f\left(t_{0}\right)-\varepsilon\right) Z_{t_{0}}\right) \cdot 1_{B_{1}^{\varepsilon}}\right) \\
& +\lim _{\varepsilon \downarrow 0} E\left(\frac{1}{\varepsilon}\left(f\left(t_{0}\right) Z_{t_{0}}-\sup _{t \neq t_{0}}|f(t)| Z_{t}\right) \cdot 1_{B_{2}^{\varepsilon}}\right) \\
& +\lim _{\varepsilon \downarrow 0} E\left(\frac{1}{\varepsilon}\left(\sup _{t \neq t_{0}}|f(t)| Z_{t}-\sup _{t \neq t_{0}}|f(t)| Z_{t}\right) \cdot 1_{B_{3}}\right) \\
= & E\left(Z_{t_{0}} \cdot 1_{B_{1}}\right)
\end{aligned}
$$

since the second summand after the second last equality vanishes by the same argument as above. The case $f\left(t_{0}\right)<0$ works similarly.

The first variation (or the partial derivatives, respectively) of a $D$-norm emerge in the easiest case of the so-called prediction problem, cf. Wang and Stoev (2011), Dombry et al. (2012) and Dombry and Éyi-Minko (2013). Suppose one knows the distribution of an SMSP $\boldsymbol{\eta}$ and the point $\left\{\eta_{t_{0}}=x\right\}, x<0$, has already been observed. We are interested in the conditional distribution of $\boldsymbol{\eta}$, given $\left\{\eta_{t_{0}}=x\right\}$. The finite-dimensional version of the following Lemma is part of Proposition 4.2 in Dombry and Évi-Minko (2013).

Lemma 4.2. Let $\boldsymbol{\eta}=\left(\eta_{t}\right)_{t \in[0,1]}$ be an SMSP with D-norm $\|\cdot\|_{D}$ generated by $\boldsymbol{Z}=\left(Z_{t}\right)_{t \in[0,1]}$. Choose an aribtrary $t_{0} \in[0,1]$. Then for every $f \in E^{-}[0,1]$ with 
$f\left(t_{0}\right)=0$ and almost all $x<0$

$P\left(\boldsymbol{\eta} \leq f \mid \eta_{t_{0}}=x\right)=\exp \left(-\left(x+\left\|f+x 1_{\left\{t_{0}\right\}}\right\|_{D}\right)\right) \cdot E\left(1_{\left\{\sup _{t \in[0,1]}|f(t)| Z_{t} \leq|x| Z_{t_{0}}\right\}} Z_{t_{0}}\right)$.

Proof. The rv $\eta_{t_{0}}$ has Lebesgue-density $e^{y}, y \leq 0$. Therefore, we have by basic rules of conditional distributions for almost all $x<0$

$$
\begin{aligned}
P\left(\boldsymbol{\eta} \leq f \mid \eta_{t_{0}}=x\right) & =\lim _{\varepsilon \downarrow 0} \frac{\varepsilon^{-1} P\left(\boldsymbol{\eta} \leq f, \eta_{t_{0}} \in(x, x+\varepsilon]\right)}{\varepsilon^{-1} P\left(\eta_{t_{0}} \in(x, x+\varepsilon]\right)} \\
& =\exp (-x) \lim _{\varepsilon \downarrow 0} \frac{P\left(\boldsymbol{\eta} \leq f, \eta_{t_{0}} \leq x+\varepsilon\right)-P\left(\boldsymbol{\eta} \leq f, \eta_{t_{0}} \leq x\right)}{\varepsilon} .
\end{aligned}
$$

Now define the function $g \in E^{-}[0,1]$ by $g(t)=f(t), t \neq t_{0}$, and $g\left(t_{0}\right)=x$. Then we have by Lemma 4.1

$$
\begin{aligned}
P\left(\boldsymbol{\eta} \leq f \mid \eta_{t_{0}}=x\right) & =\exp (-x) \lim _{\varepsilon \downarrow 0} \frac{\exp \left(-\left\|g+\varepsilon 1_{\left\{t_{0}\right\}}\right\|_{D}\right)-\exp \left(-\|g\|_{D}\right)}{\varepsilon} \\
& =-\exp (-x) \exp \left(-\|g\|_{D}\right) \cdot \nabla^{+}\|g\|_{D}\left(1_{\left\{t_{0}\right\}}\right) \\
& =\exp \left(-\left(x+\left\|f+x 1_{\left\{t_{0}\right\}}\right\|_{D}\right)\right) \cdot E\left(1_{\left\{\sup _{t \in[0,1]}|g(t)| Z_{t}=|x| Z_{t_{0}}\right\}}\right) \\
& =\exp \left(-\left(x+\left\|f+x 1_{\left\{t_{0}\right\}}\right\|_{D}\right)\right) \cdot E\left(1_{\left\{\sup _{t \in[0,1]}|f(t)| Z_{t} \leq|x| Z_{t_{0}}\right\}}\right) .
\end{aligned}
$$

The following lemma on the distribution of the increments of an SMSP can be shown by elementary calculations.

Lemma 4.3. Consider an SMSP $\boldsymbol{\eta}=\left(\eta_{t}\right)_{t \in[0,1]}$ with generator process $\boldsymbol{Z}=$ $\left(Z_{t}\right)_{t \in[0,1]}$ and choose arbitrary $s, t \in[0,1], s \neq t$. Denote by $\|\cdot\|_{D}$ the D-norm pertaining to $\left(\eta_{s}, \eta_{t}\right)$. Then for every $x \in \mathbb{R}$

$$
\begin{aligned}
& P\left(\eta_{s}-\eta_{t} \leq x\right) \\
& = \begin{cases}\int_{-\infty}^{0} \exp \left(-\|(x+y, y)\|_{D}\right) \cdot E\left(1_{\left\{y Z_{t} \leq(x+y) Z_{s}\right\}} Z_{t}\right) d y, & x<0 \\
\int_{-\infty}^{-x} \exp \left(-\|(x+y, y)\|_{D}\right) \cdot E\left(1_{\left\{y Z_{t} \leq(x+y) Z_{s}\right\}} Z_{t}\right) d y+1-\exp (-x), & x \geq 0 .\end{cases}
\end{aligned}
$$

Note that the only possible point of discontinuity of the $\operatorname{df} P\left(\eta_{s}-\eta_{t} \leq x\right)$ is $x=0$ where $P\left(\eta_{s}-\eta_{t} \leq 0\right)=\left(\|(1,1)\|_{D}\right)^{-1} E\left(1_{\left\{Z_{t} \geq Z_{s}\right\}} Z_{t}\right)$.

The preceding lemma allows us to introduce the following differentiability concept. Firstly, we call a stochastic process $\left(X_{t}\right)_{t \in[0,1]}$ almost surely differentiable in $t_{0} \in[0,1]$, if the difference quotient $\left(X_{t_{0}+h}-X_{t_{0}}\right) / h$ converges almost surely to some $\mathrm{rv} X_{t_{0}}^{\prime}$ on the real line for $h \rightarrow 0$.

Now different to that, we call a stochastic process $\left(Y_{t}\right)_{t \in[0,1]}$ differentiable in distribution in $t_{0} \in[0,1]$, if the difference quotient $\left(Y_{t_{0}+h}-Y_{t_{0}}\right) / h$ converges in distribution to some rv on the real line for $h \rightarrow 0$. 
Lastly, we call a stochastic process $\boldsymbol{\xi}=\left(\xi_{t}\right)_{t \in[0,1]}$ pathwise differentiable on $[0,1]$ if every path $\boldsymbol{\xi}(\omega)$ is differentiable on $[0,1]$.

Proposition 4.4 (Differentiability in Distribution of SMSP). Let $\boldsymbol{\eta}=\left(\eta_{t}\right)_{t \in[0,1]}$ be an SMSP with generator process $\boldsymbol{Z}=\left(Z_{t}\right)_{t \in[0,1]} \in C[0,1]$. Suppose that for some $t_{0} \in[0,1]$

$$
\frac{Z_{t_{0}+h}-Z_{t_{0}}}{h} \rightarrow h \rightarrow 0 Z_{t_{0}}^{\prime} \quad \text { a.s. }
$$

Then we have for $x \neq 0$

$$
P\left(\frac{\eta_{t_{0}+h}-\eta_{t_{0}}}{h} \leq x\right) \rightarrow_{h \rightarrow 0} H_{t_{0}}(x):=\int_{-\infty}^{0} \exp (y) E\left(1_{\left\{Z_{t_{0}}^{\prime} \leq-\frac{x}{y} Z_{t_{0}}\right\}} Z_{t_{0}}\right) d y .
$$

Proof. We have for $x \neq 0$ and $h>0$ by Lemma 4.3

$$
\begin{aligned}
& P\left(\eta_{t_{0}+h}-\eta_{t_{0}} \leq h x\right) \\
& =\int_{-\infty}^{-h|x|} \exp \left(-\|(h x+y, y)\|_{D(h)}\right) \cdot E\left(1_{\left\{y Z_{t_{0}} \leq(h x+y) Z_{t_{0}+h}\right\}} Z_{t_{0}}\right) d y+o(1)
\end{aligned}
$$

as $h \downarrow 0$, where $\|\cdot\|_{D(h)}$ is the $D$-norm generated by $\left(Z_{t_{0}+h}, Z_{t_{0}}\right)$. Now we obtain for almost all $y<-h|x|$

$$
\begin{aligned}
& E\left(1_{\left\{y Z_{t_{0}} \leq(h x+y) Z_{t_{0}+h}\right\}} Z_{t_{0}}\right) \\
& =E\left(1_{\left.\left\{y \frac{Z_{t_{0}}-Z_{t_{0}+h}}{h} \leq x Z_{t_{0}+h}\right\} Z_{t_{0}}\right)}\right. \\
& =E\left(1^{1}\left\{\frac{Z_{t_{0}+h}-Z_{t_{0}}}{h} \leq-\frac{x}{y} Z_{t_{0}+h}\right\}^{Z_{t_{0}}}\right) \\
& \rightarrow h \downarrow 0 \\
& E\left(1_{\left\{Z_{t_{0}}^{\prime} \leq-\frac{x}{y} Z_{t_{0}}\right\}} Z_{t_{0}}\right)
\end{aligned}
$$

by condition (12) which implies the assertion if $h \downarrow 0$. On the other hand, we have for $x \neq 0$ and $h<0$ by Lemma 4.3, condition (12), and the fact that $E\left(Z_{t_{0}}\right)=1$

$$
\begin{aligned}
& P\left(\eta_{t_{0}+h}-\eta_{t_{0}} \geq h x\right)=1-P\left(\eta_{t_{0}+h}-\eta_{t_{0}} \leq h x\right) \\
& =1-\int_{-\infty}^{h|x|} \exp \left(-\|(h x+y, y)\|_{D(h)}\right) \cdot E\left(1_{\left\{y Z_{t_{0}} \leq(h x+y) Z_{t_{0}+h}\right\}} Z_{t_{0}}\right) d y+o(1) \\
& \rightarrow_{h \uparrow 0} 1-\int_{-\infty}^{0} \exp (y) E\left(1_{\left\{Z_{t_{0}}^{\prime} \geq-\frac{x}{y} Z_{t_{0}}\right\}} Z_{t_{0}}\right) d y \\
& =1-\int_{-\infty}^{0} \exp (y) d y+\int_{-\infty}^{0} \exp (y) E\left(1_{\left\{Z_{t_{0}}^{\prime} \leq-\frac{x}{y} Z_{t_{0}}\right\}} Z_{t_{0}}\right) d y \\
& =\int_{-\infty}^{0} \exp (y) E\left(1_{\left\{Z_{t_{0}} \leq-\frac{x}{y} Z_{t_{0}}\right\}} Z_{t_{0}}\right) d y
\end{aligned}
$$


Proposition 4.4 gives a sufficient condition on the differentiability in distribution of an SMSP. However, it does not imply differentiability of the path of $\boldsymbol{\eta}$ at $t_{0}$. But if $\boldsymbol{\eta}$ is differentiable at $t_{0}$ a.s., then $H_{t_{0}}$ is the df of the derivative $(\partial / \partial t) \eta_{t}$ of $\boldsymbol{\eta}$ at $t=t_{0}$. We, therefore, denote by $\eta_{t_{0}}^{\prime}$ a rv which follows the df $H_{t_{0}}$.

Suppose that $\boldsymbol{Z}$ is a.s. differentiable in $t_{0}$. Then

$$
\mathbb{F}_{t_{0}}(x):=E\left(1_{\left\{Z_{t_{0}}^{\prime} \leq x Z_{t_{0}}\right\}} Z_{t_{0}}\right), \quad x \in \mathbb{R}
$$

defines a common df on $\mathbb{R}$. Denote by $\zeta_{t_{0}}$ a rv which follows this df and which is independent of $\eta_{t_{0}}$. Then we obtain the equation

$$
H_{t_{0}}(x)=P\left(-\eta_{t_{0}} \zeta_{t_{0}} \leq x\right), \quad x \in \mathbb{R}
$$

i.e., we have

$$
\eta_{t_{0}}^{\prime}={ }_{D}-\eta_{t_{0}} \zeta_{t_{0}}
$$

The pathwise derivative of $\boldsymbol{\eta}$ at $t_{0}$, if it exists, coincides, therefore, in distribution with $-\eta_{t_{0}} \zeta_{t_{0}}$.

Lemma 4.5. Suppose that $E\left(Z_{t_{0}}^{\prime}\right)$ exists. Then the mean value of $\mathbb{F}_{t_{0}}$ exists as well and coincides with $E\left(Z_{t_{0}}^{\prime}\right)$.

Proof. The expectation of an arbitrary rv $\xi$ exists iff $\int_{0}^{\infty} P(\xi>x) d x+\int_{-\infty}^{0} P(\xi<$ $x) d x<\infty$, and in this case

$$
E(\xi)=\int_{0}^{\infty} P(\xi>x) d x-\int_{-\infty}^{0} P(\xi<x) d x .
$$

As a consequence we obtain from Fubini's theorem

$$
\begin{aligned}
& \int x \mathbb{F}_{t_{0}}(d x) \\
= & \int_{0}^{\infty} 1-E\left(1_{\left\{Z_{t_{0}}^{\prime} \leq x Z_{t_{0}}\right\}} Z_{t_{0}}\right) d x-\int_{-\infty}^{0} E\left(1_{\left\{Z_{t_{0}} \leq x Z_{t_{0}}\right\}} Z_{t_{0}}\right) d x \\
= & \int_{0}^{\infty} E\left(1_{\left\{Z_{t_{0}}^{\prime}>x Z_{t_{0}}\right\}} Z_{t_{0}}\right) d x-\int_{-\infty}^{0} E\left(1_{\left\{Z_{t_{0}}^{\prime} \leq x Z_{t_{0}}\right\}} Z_{t_{0}}\right) d x \\
= & \int z \int_{0}^{\infty} 1_{\left\{z^{\prime}>x z\right\}} d x\left(P *\left(Z_{t_{0}}, Z_{t_{0}}^{\prime}\right)\right)\left(d\left(z, z^{\prime}\right)\right) \\
= & -\int z \int_{0}^{\infty} 1_{\left\{z^{\prime} \leq x z\right\}} d x\left(P *\left(Z_{t_{0}}, Z_{t_{0}}^{\prime}\right)\right)\left(d\left(z, z^{\prime}\right)\right) \\
& \quad+\int z \min \left(\frac{z^{\prime}}{z}, 0\right)\left(P *\left(Z_{t_{0}}, Z_{t_{0}}^{\prime}\right)\right)\left(d\left(z, z^{\prime}\right)\right) \\
= & \left.E\left(Z_{t_{0}}^{\prime}\right) . \quad P *\left(Z_{t_{0}}, Z_{t_{0}}^{\prime}\right)\right)\left(d\left(z, z^{\prime}\right)\right)
\end{aligned}
$$


As a consequence we obtain in particular

$$
E\left(\eta_{t_{0}}^{\prime}\right)=-E\left(\eta_{t_{0}} \zeta_{t_{0}}\right)=-E\left(\eta_{t_{0}}\right) E\left(\zeta_{t_{0}}\right)=E\left(Z_{t_{0}}^{\prime}\right)
$$

We close this section by giving some examples how Proposition 4.4 can be applied.

Example 4.6. Put

$$
Z_{t}:=U \cos ^{2}(\lambda t)+V \sin ^{2}(\lambda t), \quad t \in[0,1],
$$

where $U \geq 0, V \geq 0$ are rv with $E(U)=E(V)=1$ and $\lambda \in \mathbb{R}$. The process $\boldsymbol{Z}=\left(Z_{t}\right)_{t \in[0,1]}$ is pathwise differentiable with

$$
\frac{\partial}{\partial t} Z_{t}=\lambda \sin (2 \lambda t)(V-U)=: Z_{t}^{\prime}
$$

The distribution of the derivative in distribution $\eta_{t}^{\prime}$ is accessible under additional conditions on $U$ and $V$, but it follows immediately from Lemma 4.5 that in general $E\left(\eta_{t}^{\prime}\right)=E\left(Z_{t}^{\prime}\right)=0$.

EXAMPLE 4.7. The constant generator process $Z_{t} \equiv 1, t \in[0,1]$, gives rise to an SMSP $\boldsymbol{\eta}=\left(\eta_{t}\right)_{t \in[0,1]}$ with totally dependent univariate margins. The paths of this SMSP are constant a.s., which means that $\eta_{t}^{\prime}=0$ a.s. This fact is reflected in Proposition 4.4. If $Z_{t} \equiv 1, t \in[0,1]$, then $\mathbb{F}_{t}(x)=1_{[0, \infty)}(x)$, which implies

$$
H_{t}(x)=\int_{-\infty}^{0} \exp (y) \mathbb{F}_{t}(-x / y) d y=1_{[0, \infty)}(x) .
$$

ExAmPLE 4.8. Let $\left(Z_{0}, Z_{1}\right)$ be the generator of a bivariate standard max-stable rv $\left(\eta_{0}, \eta_{1}\right)$ with independent margins. It is well-known that such a generator has to fulfill

$$
P\left(Z_{0}=0, Z_{1}=2\right)=P\left(Z_{0}=2, Z_{1}=0\right)=1 / 2 .
$$

Now define a generator process by

$$
Z_{t}:=Z_{0}+t\left(Z_{1}-Z_{0}\right)\left(=\max \left((1-t) Z_{0}, t Z_{1}\right)\right), \quad t \in[0,1],
$$

and denote by $\boldsymbol{\eta}=\left(\eta_{t}\right)_{t \in[0,1]}$ the pertaining SMSP. Then obivously $\boldsymbol{Z}=\left(Z_{t}\right)_{t \in[0,1]}$ is pathwise differentiable with $Z_{t}^{\prime}=Z_{1}-Z_{0}, t \in[0,1]$. Lemma 4.5 instantly implies $E\left(\eta_{t}^{\prime}\right)=E\left(Z_{t}^{\prime}\right)=0$.

Furthermore, we have for $x \in \mathbb{R}$ and $t \in[0,1]$

$$
\mathbb{F}_{t}(x)=E\left(1_{\left\{Z_{t}^{\prime} \leq x Z_{t}\right\}} Z_{t}\right)= \begin{cases}1, & x \geq \frac{1}{t}, \\ 1-t, & \frac{1}{t-1} \leq x<\frac{1}{t}, \\ 0, & x<\frac{1}{t-1} .\end{cases}
$$


Hence, the corresponding $\mathrm{rv} \zeta_{t}$ that follows the df $\mathbb{F}_{t}$ is discrete with $P\left(\zeta_{t}=\frac{1}{t-1}\right)=$ $1-t$ and $P\left(\zeta_{t}=\frac{1}{t}\right)=t$. Therefore we obtain

$$
\begin{aligned}
H_{t}(x) & =\int_{-\infty}^{0} \mathbb{F}_{t}(-x / y) \exp (y) d y \\
& = \begin{cases}\int_{-\infty}^{x(1-t)} \exp (y)(1-t) d y, & x<0, \\
\int_{-\infty}^{-x t} \exp (y)(1-t) d y+\int_{-x t}^{0} \exp (y) d y, & x \geq 0\end{cases} \\
& = \begin{cases}(1-t) \exp (x(1-t)), & x<0, \\
1-t \exp (-x t), & x \geq 0 .\end{cases}
\end{aligned}
$$

EXAMPLE 4.9. Let $Z_{0} \sim \mathcal{U}(0,2)$ and $Z_{1}$ be a rv with $Z_{0}+Z_{1}=2$ a.s. Then $Z_{1} \sim \mathcal{U}(0,2)$ and $\left(Z_{0}, Z_{1}\right)$ defines a (bivariate) generator. Define the generator process

$$
Z_{t}:=Z_{0}+t\left(Z_{1}-Z_{0}\right), \quad t \in[0,1]
$$

Again, $\boldsymbol{Z}=\left(Z_{t}\right)_{t \in[0,1]}$ is pathwise differentiable with $Z_{t}^{\prime}=Z_{1}-Z_{0}, t \in[0,1]$. Clearly, $Z_{t}^{\prime} \sim \mathcal{U}(-2,2), t \in[0,1]$. This, along with the fact that $Z_{1 / 2}=1$ a.s., implies

$$
\mathbb{F}_{1 / 2}(x)=E\left(1_{\left\{Z_{1 / 2}^{\prime} \leq x\right\}}\right)=P\left(Z_{1 / 2}^{\prime} \leq x\right) .
$$

Hence, $\mathbb{F}_{1 / 2}$ is the df of the uniform distribution on $(-2,2)$. Elementary calculations now show that

$$
H_{1 / 2}(x)= \begin{cases}-\frac{x}{4} \operatorname{Ei}\left(\frac{x}{2}\right)+\frac{1}{2} \exp \left(\frac{x}{2}\right), & x<0, \\ -\frac{x}{4} \operatorname{Ei}\left(-\frac{x}{2}\right)-\frac{1}{2} \exp \left(-\frac{x}{2}\right)+1, & x>0,\end{cases}
$$

where $\operatorname{Ei}(x)=\int_{-\infty}^{x} \frac{\exp (t)}{t} d t$ denotes the exponential integral which is well-defined for $x<0$. Furthermore, we have $H_{1 / 2}(0)=1 / 2$. In particular, $H_{1 / 2}$ is continuous in 0 since the exponential integral satisfies $x \operatorname{Ei}(x) \rightarrow_{x \rightarrow 0} 0$. The density of $H_{1 / 2}$ is given by $h_{1 / 2}(x)=-\operatorname{Ei}(-|x| / 2) / 4, x \neq 0$.

\section{REFERENCES}

Aulbach, S., Bayer, V., and Falk, M. (2012). A multivariate piecing-together approach with an application to operational loss data. Bernoulli 18, 455-475. doi:10.3150/10-BEJ343

Aulbach, S., and Falk, M. (2012a). Local asymptotic normality in $\delta$ neighborhoods of standard generalized Pareto processes. J. Statist. Plann. Inference 142, 1339-1347. doi:10.1016/j.jspi.2011.12.011.

Aulbach, S., and FAlk, M. (2012b). Testing for a generalized Pareto process.

Electron. J. Stat. 6, 1779-1802. doi:10.1214/12-EJS728. 
Aulbach, S., Falk, M., and Hofmann, M. (2013). On maxstable processes and the functional D-norm. Extremes 16, 255-283. doi:10.1007/s10687-012-0160-3.

Balkema, A. A., and DE HAAn, L. (1974). Residual life time at great age. Ann. Probab. 2, 792-804. doi:10.1214/aop/1176996548.

Buishand, T. A., De HaAn, L., and Zhou, C. (2008). On spatial extremes: with application to a rainfall problem. Ann. Appl. Stat. 2, 624-642. doi:10.1214/08-A0AS159,

Deheuvels, P. (1978). Caractérisation complète des lois extrêmes multivariées et de la convergence des types extrêmes. Pub. Inst. Stat. Univ. Paris 23, 1-36.

Deheuvels, P. (1984). Probabilistic aspects of multivariate extremes. In Statistical Extremes and Applications (J. Tiago de Oliveira, ed.), 117-130. D. Reidel, Dordrecht.

Dombry, C., and ÉYi-Minko, F. (2013). Regular conditional distributions of continuous max-infinitely divisible random fields. Electron. J. Probab. 18, 1-21. doi:10.1214/EJP.v18-1991.

Dombry, C., ÉYi-Minko, F., and Ribatet, M. (2012). Conditional simulation of max-stable processes. Biometrika 100, 111-124. doi:10.1093/biomet/ass067.

Dombry, C., and Ribatet, M. (2013). Functional regular variations, pareto processes and peaks over threshold. Tech. Rep.

Einmahl, J. H. J., Krajina, A., and Segers, J. (2012). An M-estimator for tail dependence in aribtrary dimensions. Ann. Statist. 40, 1764-1793. doi:10.1214/12-AOS1023.

FAlK, M., HÜsler, J., and Reiss, R.-D. (2011). Laws of Small Numbers: Extremes and Rare Events. 3rd ed. Springer, Basel. doi:10.1007/978-3-0348-0009-9.

FALK, M., and Reiss, R.-D. (2002). A characterization of the rate of convergence in bivariate extreme value models. Statist. Probab. Lett. 59, 341-351. doi:10.1016/S0167-7152(02)00209-2.

Ferreira, A., and De HAan, L. (2012). The generalized Pareto process; with a view towards application and simulation. Tech. Rep. To appear in Bernoulli, arXiv:1203.2551v2 [math.PR].

Galambos, J. (1978). The Asymptotic Theory of Extreme Order Statistics. Wiley Series in Probability and Mathematical Statistics, 1st ed. Wiley, New York.

DE HAAn, L. (1984). A spectral representation for max-stable processes. Ann.

Probab. 12, 1194-1204. doi:10.1214/aop/1176993148 
de HaAn, L., and Ferreira, A. (2006). Extreme Value Theory: An Introduction. Springer Series in Operations Research and Financial Engineering. Springer, New York. doi:10.1007/0-387-34471-3. See http://people.few.eur.nl/ldehaan/EVTbook.correction.pdf and http://home.isa.utl.pt/ anafh/corrections.pdf for corrections and extensions.

DE HAAn, L., and Lin, T. (2001). On convergence toward an extreme value distribution in $C[0,1]$. Ann. Probab. 29, 467-483. doi:10.1214/aop/1008956340.

PICKANDS, J., III (1975). Statistical inference using extreme order statistics. Ann. Statist. 3, 119-131. doi:10.1214/aos/1176343003

Resnick, S. I., and Roy, R. (1991). Random usc functions, maxstable processes and continuous choice. Ann. Appl. Probab. 1, 267-292. doi:10.1214/aoap/1177005937.

Rootzén, H., and TAJvidi, N. (2006). Multivariate generalized Pareto distributions. Bernoulli 12, 917-930. doi:10.3150/bj/1161614952.

Wang, Y., and Stoev, S. A. (2011). Conditional sampling for spectrally discrete max-stable random fields. Adv. in Appl. Probab. 43, 461-483, http://projecteuclid.org/euclid.aap/1308662488

${ }^{1}$ University of Würzburg, Institute of Mathematics, Emil-Fischer-Str. 30,97074 WürzBurg, Germany.

E-mail address: stefan.aulbach@uni-wuerzburg.de, michael.falk@uni-wuerzburg.de, hofmann.martin@mathematik. uni-wuerzburg.de, maximilian.zott@uni-wuerzburg.de 